This document was prepared in conjunction with work accomplished under Contract No. DE-AC09-96SR18500 with the U.S. Department of Energy.

This work was prepared under an agreement with and funded by the U.S. Government. Neither the U. S. Government or its employees, nor any of its contractors, subcontractors or their employees, makes any express or implied: 1 . warranty or assumes any legal liability for the accuracy, completeness, or for the use or results of such use of any information, product, or process disclosed; or 2 . representation that such use or results of such use would not infringe privately owned rights; or 3 . endorsement or recommendation of any specifically identified commercial product, process, or service. Any views and opinions of authors expressed in this work do not necessarily state or reflect those of the United States Government, or its contractors, or subcontractors. 
WSRC-STI-2007-00614

\title{
LIGHT INDUCED TELLURIUM ENRICHMENT ON CdZnTe CRYSTAL SURFACES DETECTED BY RAMAN SPECTROSCOPY
}

\author{
Samantha A. Hawkins*, Eliel Villa-Aleman, Martine C. Duff, and Doug B. Hunter \\ Savannah River National Laboratory, Aiken, SC 29808 \\ Arnold Burger, Michael Groza, and Vladimir Buliga \\ Fisk University, Nashville, TN 37208-3051 \\ David R. Black \\ National Institute of Standards and Technology Gaithersburg, MD 20899-8520
}

${ }^{*}$ Corresponding author's phone: (803)725-5722; fax: (803)725-4704; e-mail:

Samantha.Hawkins@srnl.doe.gov

\begin{abstract}
Synthetic CdZnTe or "CZT" crystals can be grown under controlled conditions to produce high quality crystals to be used as room temperature radiation detectors. Even the best crystal growth methods result in defects, such as tellurium secondary phases, that affect the crystal's performance. In this study, CZT crystals were analyzed by micro Raman spectroscopy. The growth of Te rich areas on the surface was induced by low powered lasers. The growth was observed versus time with low power Raman scattering and was observed immediately under higher power conditions. The detector response was also measured after induced Te enrichment.
\end{abstract}

Key words: Raman spectroscopy, radiation detectors, semiconductors. 


\section{$\underline{\text { Introduction }}$}

The use of high quality crystals as room temperature gamma radiation detectors presents a significant advantage over other methods that require cryogenic cooling, high power, or larger volumes. One such crystal that has been under consideration for use as a detector is $\mathrm{Cd}_{(1-y)} \mathrm{Zn} \mathrm{n}_{\mathrm{y}} \mathrm{Te}(\mathrm{CZT})^{1-9}$. Methods for growing high quality CZT have improved significantly over the last decade ${ }^{10-13}$. As the reality of using CZT crystals as radiation spectrometers grows, so too does the necessity of having reliable and reproducible methods for measuring the quality of the crystals. Characterization techniques include infrared (IR) transmission imaging, synchrotron X-ray topography, photoluminescence spectroscopy, and Raman spectroscopy ${ }^{1-6,8,11-14}$. All of these methods have been used to test for quality to predict performance of the crystal as a gamma-ray detector. In many instances one or more of these methods have been used to test the effect a treatment, such as annealing, has on the quality of the CZT crystal $^{15-19}$.

Large localized defects can cause degraded detector performance across the entire detector ${ }^{1}$. In high quality detectors, small defects are less likely to cause a large loss in performance when a large area of the crystal is being used as the detector. However, when high spatial resolution gamma detection is desired even small defects cause a noticeable decrease in performance ${ }^{2-4,6-9}$. A common defect found in most high quality CZT crystals is Te secondary phases, often located along grain boundaries $^{1,4,8,9,19,20}$. The secondary phases can be both large inclusions (> $\left.50 \mu \mathrm{m}\right)$ and smaller precipitates $(<50 \mu \mathrm{m})$. Tellurium has a relatively small band gap, $0.33 \mathrm{eV}$, when compared with most CZT crystals, $\sim 1.6 \mathrm{eV}^{1,21}$. The Te secondary phases 
(sometimes referred to as precipitates or inclusions) distributed throughout the crystal can cause changes to the detector leakage current resulting in decreased radiation spectrometer performance ${ }^{3,4,7-9,22}$. However, it is less clear how a large area of surface Te will affect the spectrometer performance. In this study Raman spectroscopy was used to induce areas of Te secondary phases on the surfaces of the CZT crystals and the radiation spectrometer performance was measured.

\section{Experimental Method}

The CZT crystals used in this study, labeled CZT3-7-5 and CZT3-7-8, were obtained from Yinnel Tech (South Bend, IN) and were both from the same boule. The crystals were made according to the Modified Vertical Bridgman (MVB) method and have $10 \% \mathrm{Zn}^{10}$. The surfaces were polished with 0.05 micron alumina and they measured approximately $12 \mathrm{~mm} \times 12 \mathrm{~mm} \times 3.5 \mathrm{~mm}$ each for this study. Prior studies compared the performance of MVB-grown CZT crystals with polishing and chemical etching versus polishing alone ${ }^{5,23}$. The results showed that polishing alone gives a smaller full width at half maximum (FWHM) for the peaks in the gamma ray energy spectra. X-ray topography was also performed on these crystals showing the quality of their surfaces. The results for CZT3-7-8 are shown in a previous paper ${ }^{5}$. The X-ray topography measurements were performed at the Advanced Photon Source at Argonne National Laboratory (Argonne, IL) using beam line 33-BM. The incident energy of $9 \mathrm{keV}$ was used from the white radiation source using a Si(111) double crystal monochromator. A rotating foam disc was placed between the sample and the monochromator to serve as a random phase object. This removed structure in the 
incident beam due to phase contrast resulting from imperfections in the beam line $\mathrm{Be}$ windows. The face of CZT3-7-5 was parallel to the (111) surface and a symmetric (333) image was recorded with the diffraction vector pointing out of the image. The image was documented on high contrast lithographic film that was positioned parallel to the sample. This position was used to avoid image foreshortening. CZT3-7-8 had a high quality surface as shown by X-ray topography and also was a high performer as a gamma radiation spectrometer ${ }^{5}$.

Micro-Raman spectroscopy was performed using a Jobin Yvon LabRAM HR800-UV Raman microscope with a 50X objective and a cooled CCD camera detector. The micro-Raman spectra were collected using an Argon ion laser. The scattering light used during data collection was attenuated using filters with resulting beam power from $\sim 2 \mu \mathrm{W}$ to $20 \mathrm{~mW}$. The Raman system has an automated microscope stage with mapping capability which was used to probe the crystal labeled CZT3-7-8 for uniformity, surface contaminants, and the Te precipitates. Additionally, the attenuated doubled light from a Spectra Physics Nd:YAG laser was used to expose larger areas of the crystal labeled CZT3-7-5. After the exposure to $532 \mathrm{~nm}$ light the gamma radiation detector response measurements were also made on CZT3-7-5. The method for detector response measurements used was described in detail in a previous paper ${ }^{5}$.

\section{$\underline{\text { Results and Discussion }}$}

The X-ray topography image of CZT3-7-5 is shown in figure 1 . This image shows a number of defects present in the crystal. The X-ray topography image of CZT3-7-8, shown in a previous paper, had very few defects ${ }^{5}$. The corresponding 
gamma radiation detector response measurements for this material confirmed that it was a high quality performer with a FWHM of $1.7 \%$ for the $662 \mathrm{keV}$ peak of ${ }^{137} \mathrm{Cs}$. The X-ray topography image of highly polished CZT3-7-5 shows that the crystalline quality of this material is lower than that of CZT3-7-8. Due to its lower crystalline quality, we anticipated that this material may have a lower gamma radiation spectrometer performance which was confirmed with measurements using an ${ }^{241} \mathrm{Am}$ source (shown in figure 2a). Current-voltage measurements of CZT3-7-5 (figure 2b) demonstrate that even though this crystal has some defects, it a moderate performer with a high bulk resistivity.

CZT3-7-8 was exposed to the $514 \mathrm{~nm}$ radiation from an Argon ion laser with $\sim 20$ $\mathrm{mW}$ power and had very intense peaks at $124 \mathrm{~cm}^{-1}$ and $144 \mathrm{~cm}^{-1}$ and a much less intense, broad peak centered $\sim 160 \mathrm{~cm}^{-1}$ in the Raman shift spectrum. These peaks were consistent with those previously reported for high quality CZT crystals ${ }^{13,15-18}$. However, in different locations on the crystal, the $124 \mathrm{~cm}^{-1}$ and $144 \mathrm{~cm}^{-1}$ peaks were much less intense when lower laser power was used. When the laser power was attenuated to below $100 \mu \mathrm{W}$ these peaks were near background. The intensity of these two peaks was highly dependant on the laser power and duration of exposure.

The peaks at $124 \mathrm{~cm}^{-1}, \sim 140 \mathrm{~cm}^{-1}$ and $\sim 160 \mathrm{~cm}^{-1}$ have been attributed to surface Te precipitates, the transverse optical (TO) mode of $\mathrm{CdTe}$, and the longitudinal optic (LO) mode of CdTe respectively ${ }^{13,15-18,21}$. Figure 3 shows the growth of the $124 \mathrm{~cm}^{-1}$ and $144 \mathrm{~cm}^{-1}$ peak intensity as a function of time with low power laser exposure. The growth of the peak at $124 \mathrm{~cm}^{-1}$ is not infinite as can be seen in figure 4 . A plot of the intensity of this peak versus time shows that the growth slows down and appears to 
stop after approximately one hour. The peaks at $\sim 120 \mathrm{~cm}^{-1}$ and $\sim 140 \mathrm{~cm}^{-1}$ are in the same peak shift positions and exhibit the same behavior that was noted when crystalline Te was melted and became an amorphous phase ${ }^{21}$. These peaks became sharper and grew in at approximately equivalent intensities. This observation indicates that a significant change occurred on the CZT surface in the possible form of amorphous Te growth. It also indicates that the peak at $144 \mathrm{~cm}^{-1}$ that was previously attributed to a CdTe mode on the CZT surface is more likely to be due to Te secondary phases.

A Raman intensity map of an area of CZT3-7-8 is shown in figure 5. The map is of the $124 \mathrm{~cm}^{-1}$ peak intensity using a laser power less than $60 \mu \mathrm{W}$ and short exposure time. The three bright spots are areas where Raman spectra with a slightly higher laser power had already been taken. Thus, the change in the surface caused by the Raman laser is permanent and is easily accomplished. The figure also shows the corresponding Raman spectra from different areas of the map. The bright spot has relatively more intense peaks at both $124 \mathrm{~cm}^{-1}$ and $144 \mathrm{~cm}^{-1}$, where the dark area has very low intensity peaks at both of those Raman shifts.

An area of CZT3-7-5 was exposed to a low power doubled Nd:YAG laser to induce Te enrichment on the surface. The crystal was exposed in one corner on the Te terminating face and the opposite corner on the Cd terminating face. Figure 6 shows Raman spectra of the area of the crystal that was exposed, before and after the laser exposure with both 30 second and 300 second collection time. Both crystal faces produces identical Raman spectra both before and after the laser exposure. These spectra were collected using an argon ion laser attenuated to approximately $240 \mu \mathrm{W}$ 
laser power. Figure 6d) shows that even with such a low power laser, a 300 second exposure is sufficient to cause growth of the $124 \mathrm{~cm}^{-1}$ and $144 \mathrm{~cm}^{-1}$ peaks. The surface was probed in different areas prior to the Nd:YAG laser exposure in order to ensure that the surface did not already contain areas of high Te content. It is likely that very small Te precipitates were already present, however none were found with random area sampling. After the surface was exposed with a single pulse from the Nd:YAG laser the surface was again probed by Raman spectroscopy. The exposed area exhibited a considerable increase in intensity of the 124 and $144 \mathrm{~cm}^{-1}$ peaks. No other changes in the Raman spectra were observed. These results indicate that Te aggregation and possibly CdTe bond dissolution were the only changes induced by the laser and detected by Raman spectroscopy. The unexposed area of the surface was also probed in several random spots to confirm that no changes were induced elsewhere on the crystal.

The crystal resisitivity and spectrometer performance was then re-measured. Figure 7 demonstrates that the detector response, with the gold anode on the $\mathrm{Cd}$ rich face, decreased noticeably from FWHM $=8.2 \%$ on the polished reference surface to $11.7 \%$ at the laser exposed area. No gamma detector response could be measured on the Te rich face due to excessive electronic noise. Thus, laser exposure on this side was highly damaging to the CZT crystal's use as a gamma spectrometer. The resistivity of the laser exposed area on the Cd rich face was only slightly affected. However, the resisitvity of the laser exposed spot on the Te rich face decreased by approximately a factor of 3 thus changing the leakage current and reducing the crystal's ability to be used as a gamma spectrometer. 


\section{Conclusions}

With a low laser power, (potentially) amorphous Te enriched areas can be induced on a CZT crystal surface. The change in the surface is irreversible and can be confirmed with Raman spectroscopy without further damage by using an ultra-low power laser beam with short exposure time. The peaks at $124 \mathrm{~cm}^{-1}$ and $144 \mathrm{~cm}^{-1}$ in the Raman shift spectrum both grow with increased laser exposure and it is likely that they are both due to Te secondary phase growth. Although the exposure reduces the performance on the $\mathrm{Cd}$ terminating face, the effect of the laser exposure is more profound on the Te terminating face of CZT3-7-5. This is likely due to the greater amount of Te present on this Te rich side of the CZT.

Acknowledgement: This work was supported by US DOE - National Nuclear Security Administration, through the office of Nonproliferation Research and Development (NA22). Work by Fisk University was also supported by the National Science Foundation through the Fisk University Center for Physics and Chemistry of Materials (CPCoM), Cooperative Agreement CA: HRD-0420516 (CREST program) and through US DOE NA-22, Grant No. DE-FG52-05NA27035. 


\section{$\underline{\text { References }}$}

${ }^{1}$ T.E. Schlesinger, J.E. Toney, H. Yoon, E.Y. Lee, B.A. Brunett, L. Franks, R.B. James, Mat. Sci. Eng. R v32, 103 (2001).

${ }^{2}$ B.A. Brunett, J.M. Van Scyoc, N.R. Hilton, J.C. Lund, R.B. James, T.E. Schlesinger, IEEE T. Nucl. Sci. v46, 237 (1999).

${ }^{3}$ B.A. Brunett, J.M. Van Scyoc, T.E. Schlesinger, R.B. James, Nucl. Instrum. Meth. A v458, 76 (2001).

${ }^{4}$ P.N. Luke, M. Amman, J.S. Lee, IEEE T. Nucl. Sci., v51, 1199 (2004).

${ }^{5}$ M.C. Duff, D.B. Hunter, A. Burger, M. Groza, V. Buliga, D.R. Black, Appl. Surf. Sci, In press (2008).

${ }^{6}$ A. Burger, K. Chattopadhyay, H. Chen, X. Ma, J.-O. Ndap, M. Schieber, T.E.

Schlesinger, H.W. Yao, J. Erickson, R.B. James, Nucl. Instrum. Meth. A v448, 586 (2000).

${ }^{7}$ G.A. Carini, A.E. Bolotnikov, G.S. Camarda, G.W. Wright, R.B. James, Appl. Phys. Lett. v88, 143515 (2006).

${ }^{8}$ A.E. Bolotnikov, G.S. Camarda, G.A. Carini, Y. Cui, L. Li, R.B. James, Nucl. Instrum. Meth. A v579, 125 (2007).

${ }^{9}$ A.E. Bolotnikov, G.S. Camarda, G.A. Carini, Y. Cui, L. Li, R.B. James, Nucl. Instrum. Meth. A v571, 687 (2007).

${ }^{10}$ L. Li, F. Lu, K. Shah, M. Squillante, L. Cirinano, W. Yao, R.W. Olson, P. Luke, Y. Nemirovsky, A. Burger, G. Wright, R.B. James, Nuclear Sci. Symp. Conf. Rec. IEEE. V4, 2396 (2001). 
${ }^{11}$ M. Schieber, R.B. James, H. Hermon, A. Vilensky, I. Baydjanov, M. Goorsky, T. Lam, E. Meerson, H.W. Yao, J. Erickson, E. Cross, A. Burger, J.O. Ndap, G. Wright, M.

Fiederle, J. Cryst. Growth v231, 235 (2001).

${ }^{12}$ C.M.Greaves, B.A. Brunett, J.M. Van Scyoc, T.E. Schlesinger, R.B. James, Nucl. Instrum. Meth. A v458, 96 (2001).

${ }^{13}$ M. Sridharan, Sa.K. Narayandass, D. Mangalaraj, H. Chul Lee, Vacuum v70, 511 (2003).

${ }^{14}$ T. Wang, W. Jie, J. Zhang, G. Yang, D. Zeng, Y. Xu, S. Ma, H. Hua, K. He, J. Cryst. Growth v304, 313 (2007).

${ }^{15}$ H. Huang, J. Xu, J. Wang, C. Zhang, Y. Mo, S. Pan, G. Zhang, Materials for Infrared Detectors, R.E. Longshore, Editor, Proc. SPIE v4454, 244 (2001).

${ }^{16}$ K. Prabakar, S. Venkatachalam, Y.L. Jeyachandran, Sa.K. Narayandass, D. Mangalaraj, Mat. Sci. Eng. B-Solid v107, 99 (2004).

${ }^{17}$ M.G. Sridharan, M. Mekaladevi, Sa.K. Narayandass, D. Mangalaraj, H. Chul Lee, J. Optoelectron. Adv. M. v7, 1479 (2005).

${ }^{18}$ M.G. Sridharan, Sa.K. Narayandass, H. Chul Lee, J. Optoelectron. Adv. M. v7, 1483 (2005).

${ }^{19}$ D. Zeng, W. Jie, G. Zha, T. Wang, G. Yang, J. Cryst. Growth In press (2007).

${ }^{20}$ T. Wang, W. Jie, D. Zeng, Mat. Sci. Eng. A-Struct. In press (2007).

${ }^{21}$ A.S. Pine, G. Dresselhaus, Phys. Rev. B v4, 356 (1971).

${ }^{22}$ P. Rudolph, M. Mühlberg, Mat. Sci. Eng. B v16, 8 (1993).

${ }^{23}$ M.C. Duff, D.B. Hunter, P. Nuessle, D.R. Black, H. Burdette, J. Woicik, A. Burger, M. Groza, J. Elec. Mat. V36, 1092 (2007). 
WSRC-STI-2007-00614

Figure1

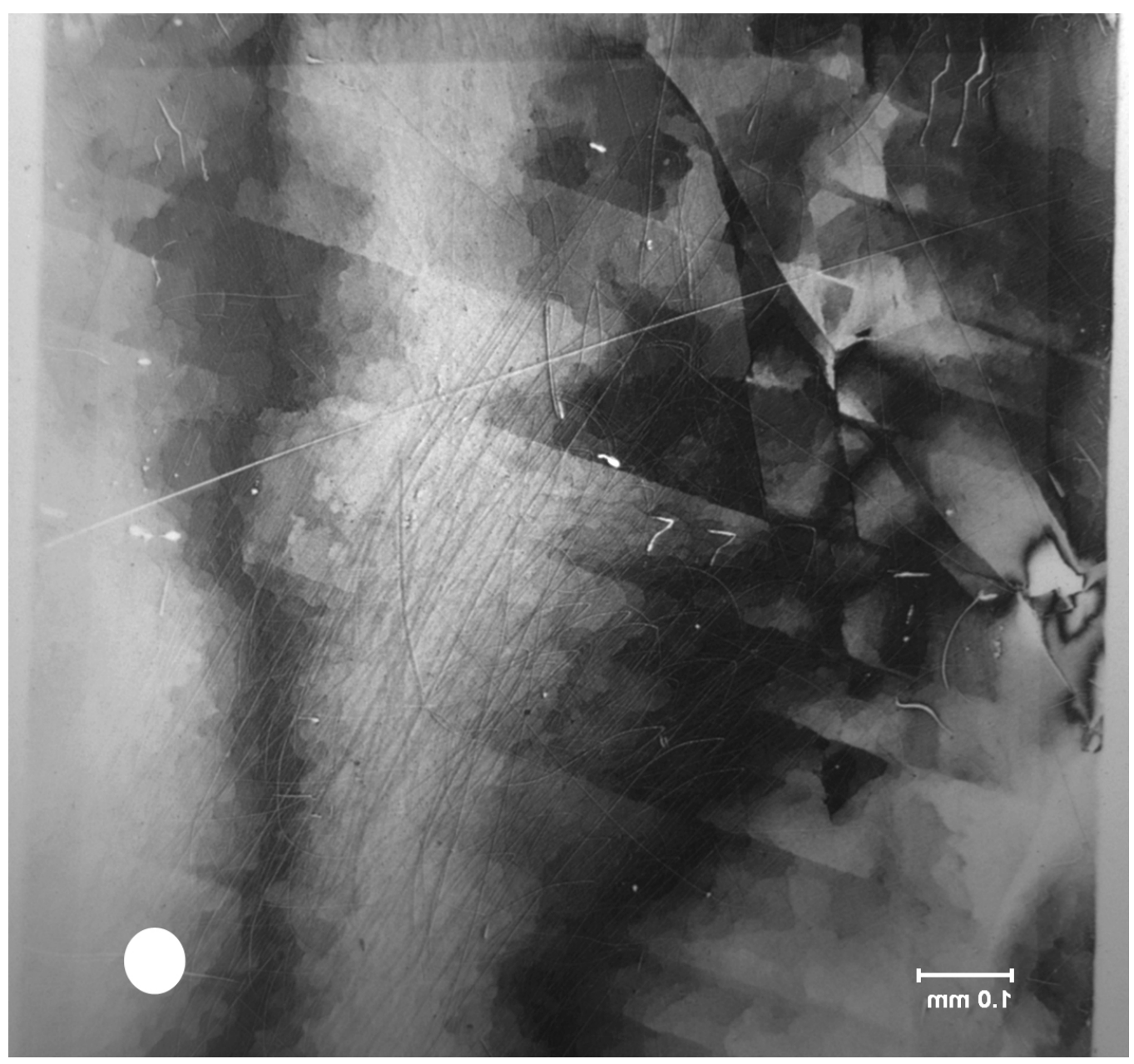


Figure 2
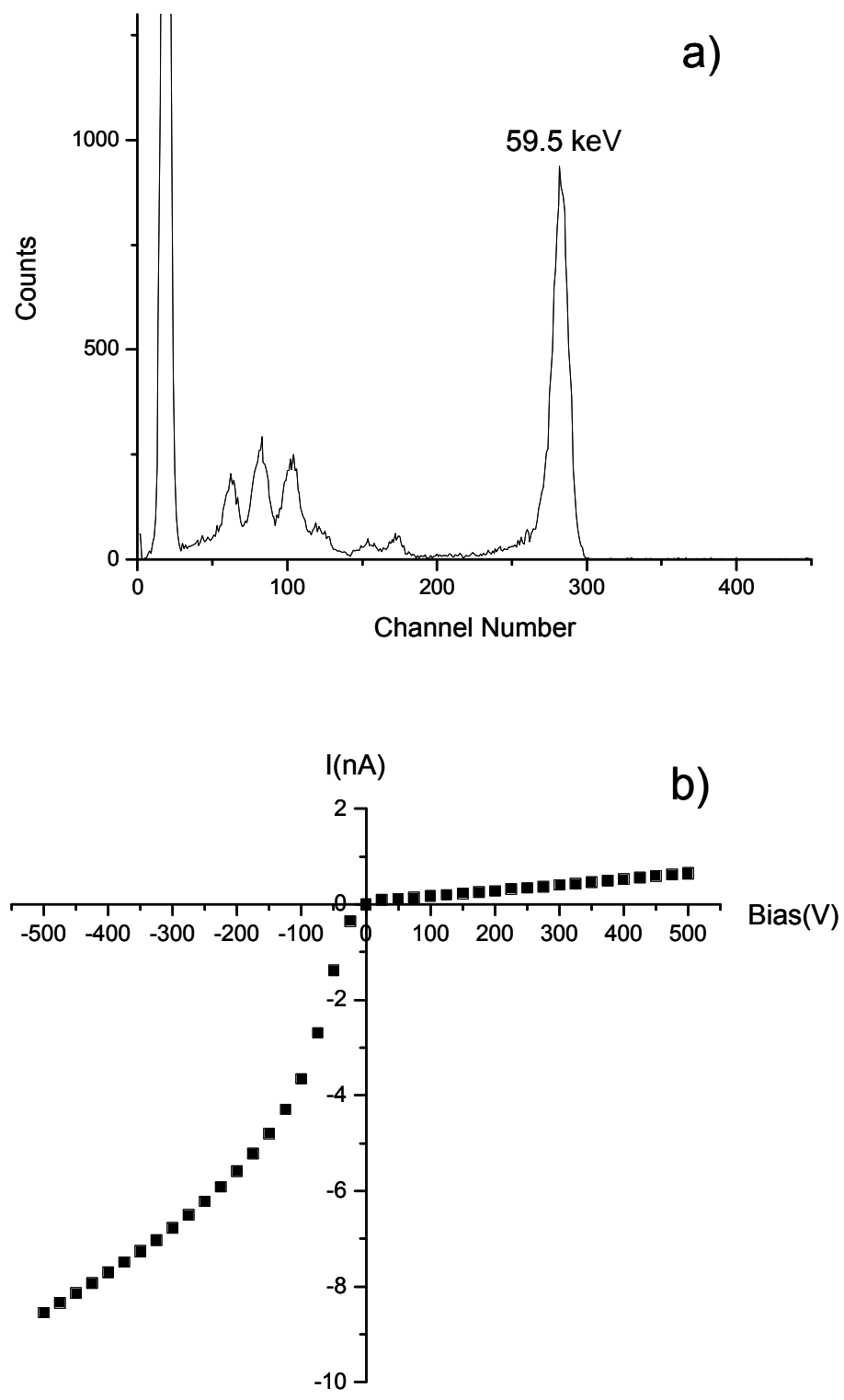
Figure 3

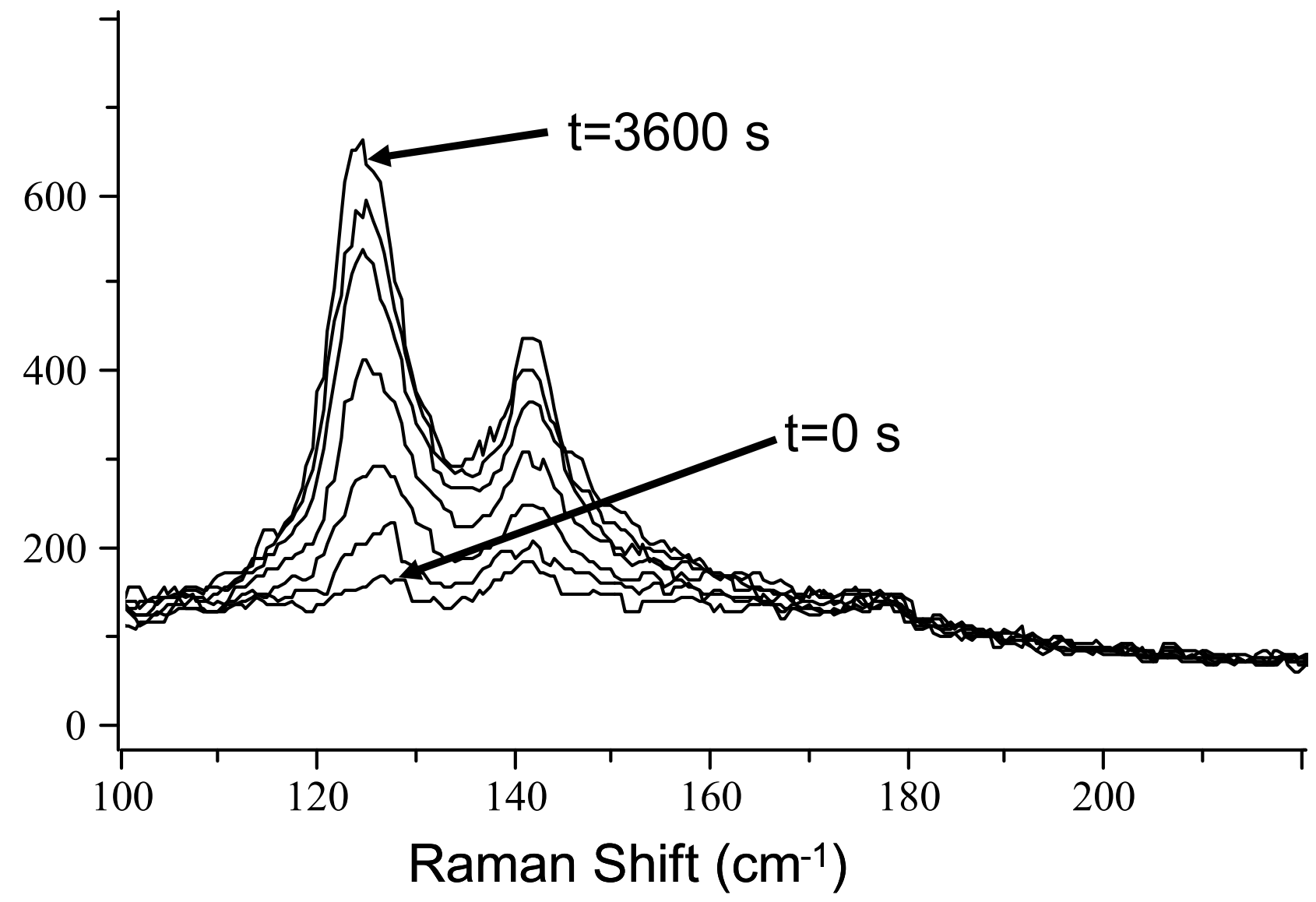


Figure 4

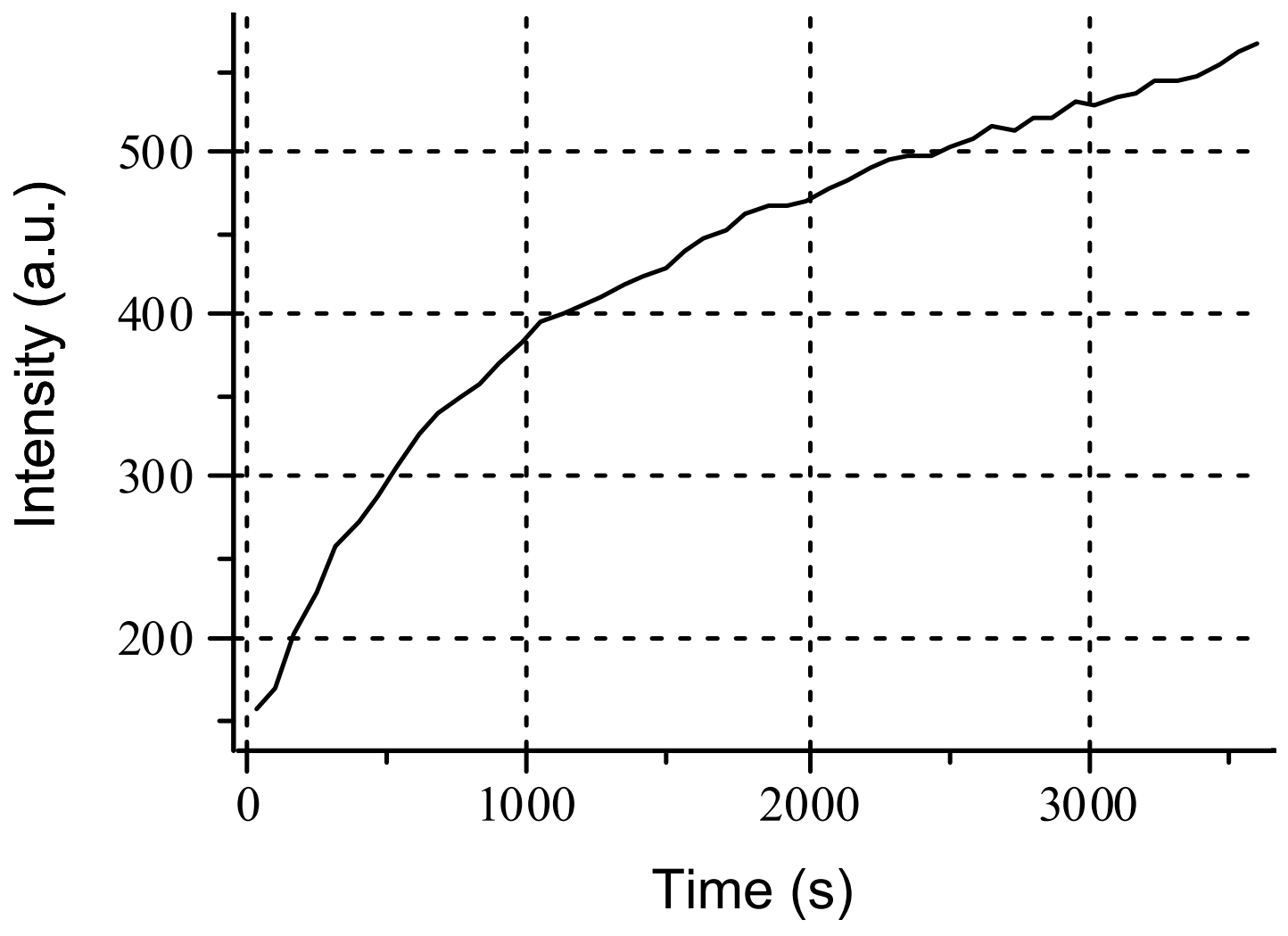


Figure 5

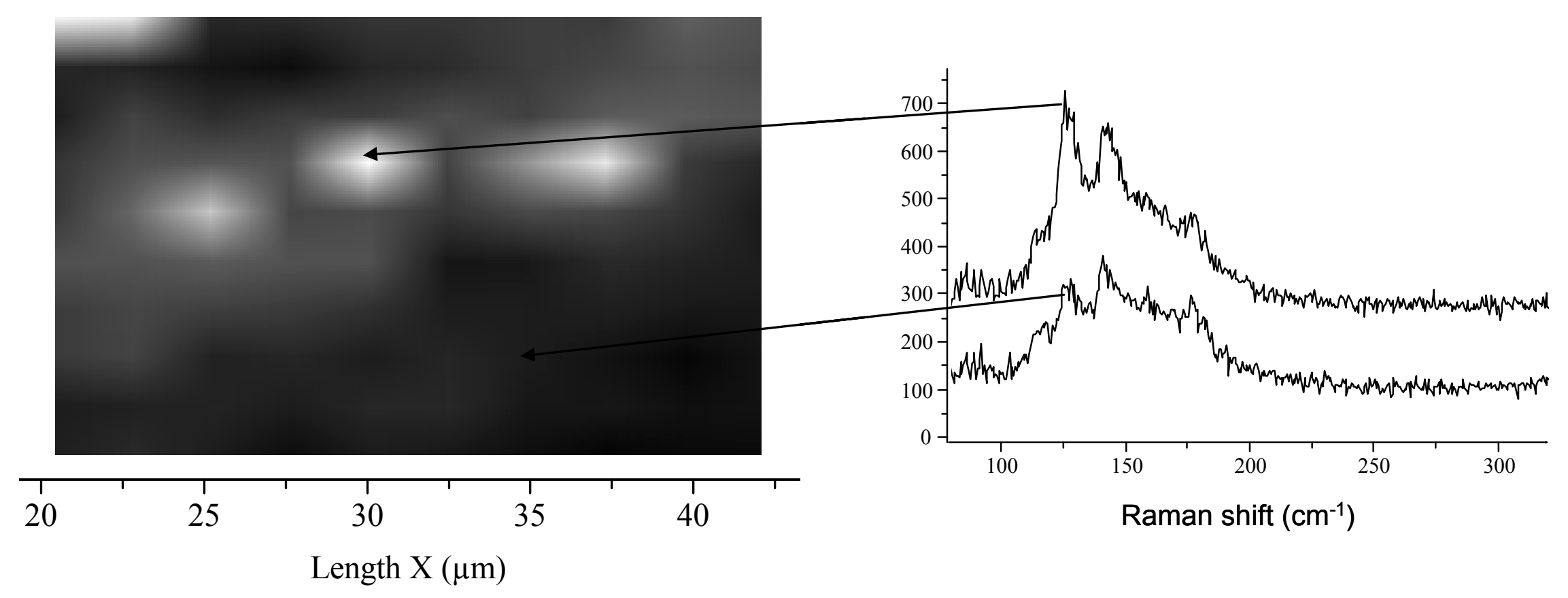


Figure 6
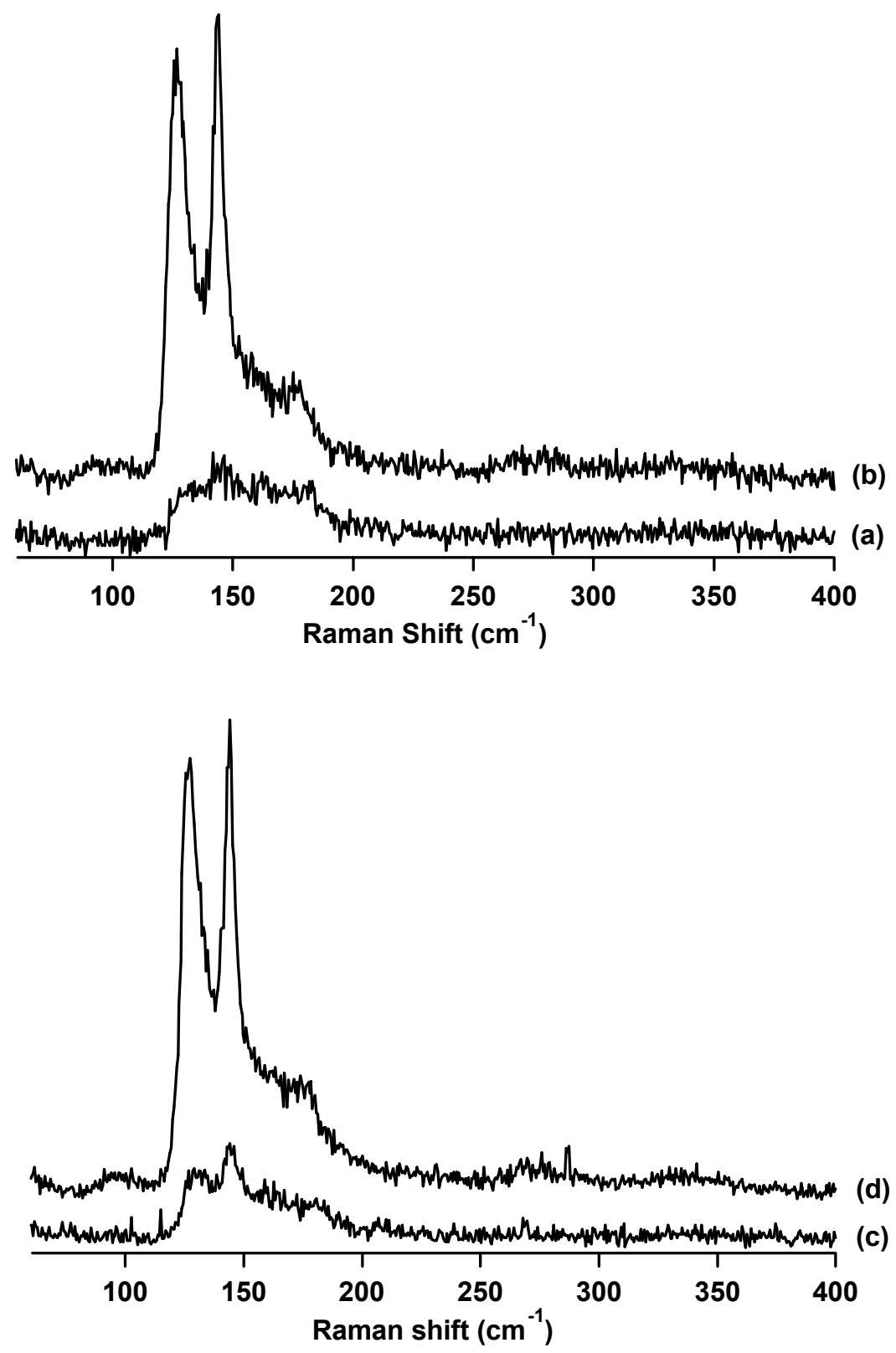
Figure 7

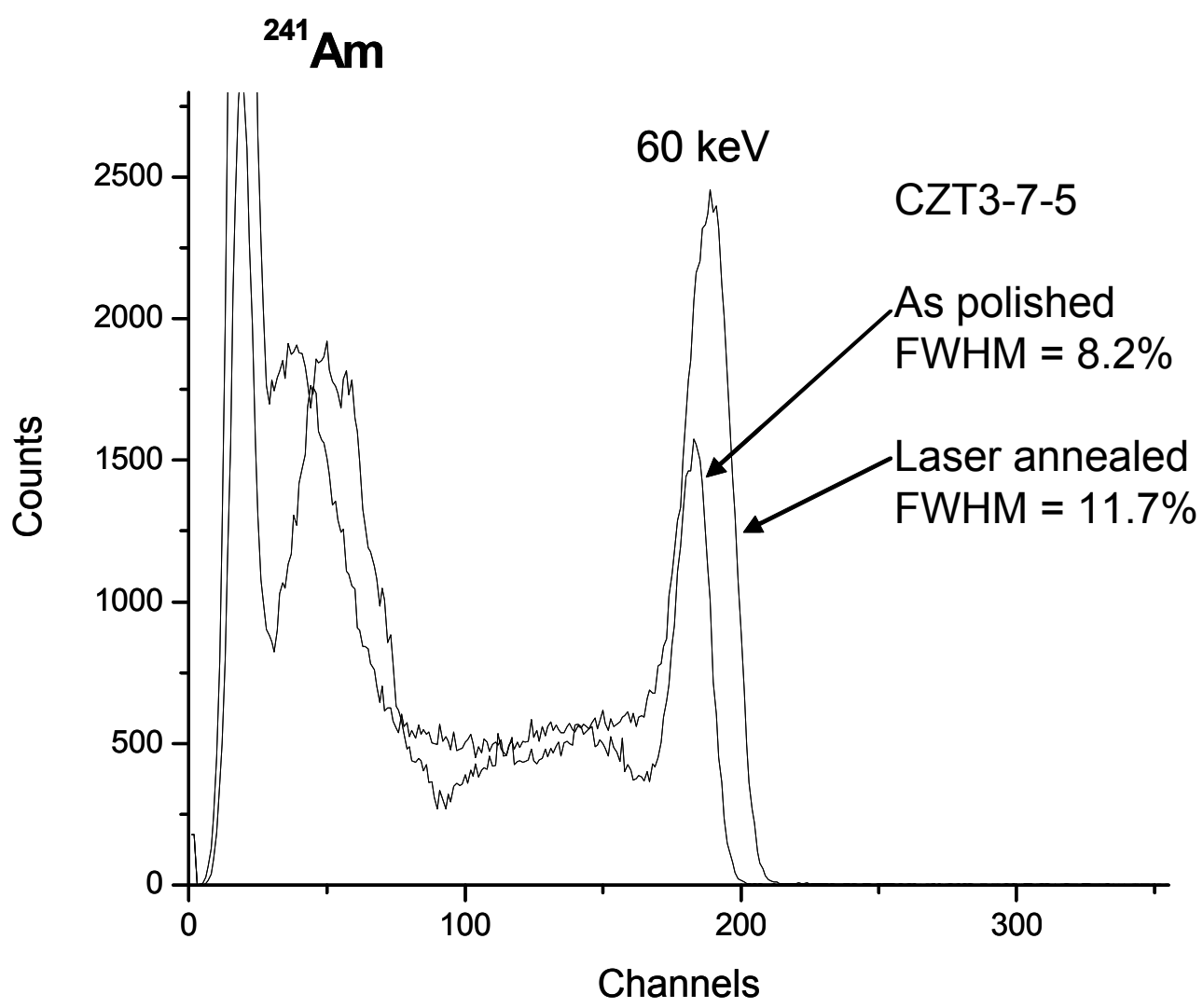




\section{Figure captions}

Figure 1: CZT3-7-5 (333) X-ray topography image. The image was recorded with 2 exposures, $\Delta \theta=0.05^{\circ}$.

Figure 2: CZT3-7-5, as polished with gold contacts on the surface a) gamma detector response measurement with ${ }^{241} \mathrm{Am}$ source, $500 \mathrm{~V}, 1 \mu \mathrm{sec} ; \mathrm{FWHM}=8.2 \%$ at $59.54 \mathrm{keV}$ b) current-voltage measurement, $\rho=2.3 \times 10^{10} \Omega \mathrm{cm}$

Figure 3: Raman spectra were collected versus laser exposure time on CZT3-7-8. The spectra shown range from initial exposure to 3600 seconds of laser exposure. The laser power used was 60 microwatts.

Figure 4: The intensity of the Raman peak at $124 \mathrm{~cm}^{-1}$ versus time of laser $(514 \mathrm{~nm}, 60$ microwatts) exposure on crystal CZT3-7-8.

Figure 5: Map of an area of CZT3-7-8. The bright spots are areas where Raman spectra had been taken using higher than 100 microwatt laser power. The map was collected using approximately 20 microwatts from an Argon ion laser with low exposure time. The Raman spectra corresponding to the bright area and dark area of the map are shown on the right.

Figure 6: CZT3-7-5 Raman spectra collected with an Argon ion laser with 240 microwatts power; a) and b) were collected with $30 \mathrm{sec}$ total Raman laser exposure 
time, c) and d) were collected with 300 seconds Raman laser exposure time: a) and c) before exposure to the Nd:YAG laser and b) and d) after exposure to the Nd:YAG laser. The beam size of the Nd:YAG laser was approximately $2 \mathrm{~mm}$ diameter.

Figure 7: CZT3-7-5 Cd rich face gamma detector response measurement with ${ }^{241} \mathrm{Am}$ source, $3 \mathrm{~mm}$ gold contacts used as anodes: as polished reference surface $\mathrm{FWHM}=$ $8.2 \%$ and laser annealed spot FWHM $=11.7 \%$ at $59.54 \mathrm{keV}$ 